\title{
De l'utopie à la praxis : William Morris et le Guild Socialism
}

From Utopia to Praxis: William Morris and Guild Socialism

\section{Valérie Auda-André}

\section{(2) OpenEdition}

12 Journals

\section{Édition électronique}

URL : http://journals.openedition.org/rfcb/3286

DOI : $10.4000 / \mathrm{rfcb} .3286$

ISSN : 2429-4373

\section{Éditeur}

CRECIB - Centre de recherche et d'études en civilisation britannique

\section{Édition imprimée}

Date de publication : 1 septembre 2004

ISBN : 2-911580-19-2

ISSN : 0248-9015

\section{Référence électronique}

Valérie Auda-André, «De l'utopie à la praxis : William Morris et le Guild Socialism », Revue Française de Civilisation Britannique [En ligne], XIII-1 | 2004, mis en ligne le 01 septembre 2004, consulté le 07 octobre 2019. URL : http://journals.openedition.org/rfcb/3286 ; DOI : 10.4000/rfcb.3286

Ce document a été généré automatiquement le 7 octobre 2019.

\section{cc)}

Revue française de civilisation britannique est mis à disposition selon les termes de la licence Creative Commons Attribution - Pas d'Utilisation Commerciale - Pas de Modification 4.0 International. 


\title{
De l'utopie à la praxis : William Morris et le Guild Socialism
}

\author{
From Utopia to Praxis: William Morris and Guild Socialism
}

\author{
Valérie Auda-André
}

1 Comment évaluer la contribution des Nouvelles de Nulle Part de William Morris à l'émergence d'une pensée socialiste en Grande-Bretagne ? À l'évidence, la question est complexe. Traditionnellement, l'évaluation de la portée de la pensée morrissienne est indissociable de la question de sa conformité avec le dogme marxiste. Il s'agit certes là d'une problématique incontournable, ayant du reste longtemps dominé les études morrissiennes, peut-être au détriment d'autres aspects de l'œuvre de William Morris. Pour la critique marxiste, le choix de l'utopie comme mode d'expression demeure un choix déconcertant. E.P. Thompson rappelle la fraîcheur de l'accueil que Friedrich Engels avait réservé à News from Nowhere ${ }^{1}$. Ce dernier s'irritait de trouver dans le texte de Morris les échos passéistes des utopies socialistes qu'il pensait avoir contribué à déboulonner comme autant de vieilles idoles. D'aucuns ont voulu voir dans Nouvelles de Nulle Part une tentative de naturalisation plus ou moins réussie de la pensée de Karl Marx et ont fait violence au texte en ne l'évaluant qu'au regard de sa conformité avec l'orthodoxie ${ }^{2}$. La critique d'obédience marxiste est irrésistiblement tentée de mesurer l'importance de News from Nowhere à l'aune de sa conformité avec la pensée de l'auteur du Capital ; lorsque le texte résiste à l'assimilation, la tentation est grande de suppléer les manques ou les absences en effectuant un détour par d'autres écrits de Morris, à la forme plus canonique, qui permettent de proposer au lecteur un News from Nowhere fécondé par la pensée marxiste ${ }^{3}$.

2 Parallèlement à cet intertexte marxiste, Nouvelles de Nulle Part est traversé par différents courants de pensée parmi lesquels le lecteur décèle en filigrane des échos de la critique « romantique » du capitalisme industriel élaborée notamment par Carlyle et Ruskin, des résurgences du discours des penseurs proto-socialistes du début du siècle, radicaux, chartistes ou owénistes, des parallèles avec les analyses des anarchistes tels que Kropotkine. William Morris est sans nul doute l'héritier d'une riche tradition, dont la réflexion s'est nourrie de nombreux échanges avec ceux de ses contemporains qui 
s'étaient engagés sur la voie de la contestation de l'ordre établi; dans News from Nowhere, ces influences se conjuguent pour présider à l'élaboration d'un monde idéal dont l'une des fonctions est bien sûr la dénonciation de la société victorienne. Or, si la question se pose des influences perceptibles dans l'œuvre de William Morris, et notamment dans ses Nouvelles de Nulle Part, il paraît également intéressant de s'interroger sur les prolongements de la pensée morrissienne et sur son impact à la fois sur l'évolution du socialisme en Grande-Bretagne et sur les orientations du mouvement qui allait tenter d'incarner l'idéal socialiste, le mouvement travailliste.

C'est donc grâce à l'étude des modalités de l'utopie explorées et exploitées dans News from Nowhere, ainsi qu'à l'analyse des conflits qui ont opposé Morris à ses contemporains et des convergences perceptibles dans les travaux des Guild Socialists, qu'il est possible de tenter d'identifier la spécificité du discours morrissien et d'évaluer sa contribution à l'histoire du socialisme britannique.

\section{Les voies de l'utopie}

$4 \quad$ Nouvelles de Nulle Part s'inscrit d'emblée dans la tradition de la pensée utopique. Le titre de l'ouvrage publié en 1890 est sans équivoque, et revendique avec une simplicité désarmante sa filiation avec les grandes utopies, à commencer par l'Utopia (1516) de Thomas More. La simplicité du titre choisi par Morris n'est pourtant qu'apparente ; certes, l'auteur s'y réclame d'un genre aux codes bien établis, mais il introduit d'emblée une impasse logique en plaçant dans une relation de proximité deux termes apparemment contradictoires : il est malaisé de concevoir, dans un ordre rationnel, des «nouvelles de nulle part ». News from Nowhere introduit un élément de discontinuité avec le réel rationnel et suggère la nécessité de ce que l'on pourrait appeler suspension of disbelief, terme qui décrit l'attitude caractéristique du lecteur/spectateur face à une œuvre de fiction, lorsque celui-ci s'abandonne au récit. Le dispositif narratif mis en place par l'auteur transgresse les codes réalistes par le biais du procédé de la « double scène ", lorsque le protagoniste franchit la frontière entre réel imaginaire, présent et avenir. La fin du récit est à cet égard fort éclairante, qui voit William Guest faire le voyage à l'envers pour rejoindre le présent : le monde parfait de Nulle Part se fige alors en une image fixe pour redevenir objet de contemplation, une gravure, un vitrail ou une toile sur laquelle l'artiste projette son désir.

5 Toutefois, il paraît également judicieux de souligner les convergences entre les deux termes juxtaposés dans le titre de l'œuvre de Morris : news et nowhere se font écho dans l'évocation d'un ailleurs qui reste à construire, d'un nulle part qui invite à l'exploration et qui redouble l'idée de nouveauté contenue dans le premier terme du titre. News from Nowhere apparaît alors comme un titre sursignifié s'inscrivant dans une stratégie de saturation. Dans une perspective plus politique ou idéologique, on peut y percevoir une forme de revendication de la part d'un auteur qui affiche clairement sa volonté de ne pas limiter l'éventail des possibles. Dans la quête morrissienne, le socialisme ouvre une ère nouvelle, synonyme d'émancipation des contraintes et conventions de l'ordre bourgeois, le choix formel de l'utopie signalant ainsi une volonté d'établir une distance entre News from Nowhere et les normes esthétiques contemporaines. Il s'agit également de revendiquer la liberté d'imaginer un monde nouveau, en dehors des contraintes doctrinales. Le monde de Nowhere est aussi un « nulle part » idéologique qui privilégie 
une conception ouverte de la nouvelle société, à la fois terra incognita (Morris se refuse à nommer le nouveau monde) et terra nullius qui se situe en deçà ou au delà de l'idéologie.

On peut relever dans Nouvelles de Nulle Part la présence de plusieurs modalités utopiques dont la coexistence renforce le propos de l'auteur. Le Nulle Part de William Morris revêt tout d'abord une dimension arcadienne incontestable : Nulle Part est un «Paysque-le-cœur désire $\rrbracket^{4}$, un monde parfait, Éden terrestre baigné de la douce lumière d'un mois de juin qui contient la promesse d'un bonheur sans faille ; l'impression qui domine est celle de plénitude, d'harmonie et de sérénité dans un monde qui paraît avoir été soustrait aux conséquences du péché originel. Nulle Part semble être un monde d'après la rédemption: cet objet du désir est sans désir, la nature y est généreuse et bienveillante, sans pour autant qu'il y soit question d'abondance ou de profusion, les relations sociales s'y établissent sur le mode de la fraternité, les hommes y jouissent avec sérénité du bonheur d'exister.

7 La présence d'un mythe arcadien au cœur de News from Nowhere ne suffit certes pas à rendre compte du caractère utopique de l'œuvre. Le nouveau monde qui prend vie sous les yeux du lecteur est également investi d'une dimension critique. La dénonciation est l'une des fonctions traditionnelles de l'utopie et le procédé de mise en regard des deux mondes, monde réel et monde rêvé, société contemporaine et société du futur, invite la comparaison. Raymond Williams écrit à propos de l'utopie : 'what most has to be looked at, in properly utopian or dystopian fiction, is the continuity, the implied connection, which the form is intended to embody. ${ }^{5}$ On remarquera que Nouvelles de Nulle Part place la question de la relation entre réel et imaginaire, présent et avenir, au coeur de son dispositif narratif et que, de plus, le lien entre les deux mondes n'a rien d'implicite : il est assuré par la présence physique de la figure du passeur, William Guest, qui jette un pont entre les rives des deux univers; par ailleurs, les notations abondent qui signifient non pas la coexistence impossible de ces deux univers mais l'existence d'un lien quasi généalogique qui les relie; enfin, c'est toute l'ambiguïté de "nulle part », univers à la fois étrange et familier, radicalement différent et pourtant reconnaissable, qui suggère l'invraisemblable contiguité de ces deux mondes.

Cette étrange proximité favorise bien sûr la comparaison et incite à la dénonciation de l'univers dystopique de l'Angleterre victorienne. Le contraste qui s'établit entre «ici » et «nulle part» désigne l'Angleterre du XIX siècle comme un univers dégradé. Les préoccupations artistiques de William Morris ressurgissent dans son évocation en contrepoint d'une société dont la conception du Beau est incontestablement corrompue. Le spectre des notations esthétiques balayé par Morris est extrêmement large: les jugements portés par l'auteur concernent le domaine architectural (les bâtiments publics, les ponts), l'habitat (à la fois les habitations insalubres du prolétariat ouvrier mais aussi les riches demeures bourgeoises), l'absence de réflexion menée sur l'aménagement de l'espace urbain dont le développement anarchique reflète à la fois les fractures d'une société de classes, les impératifs de l'économie de marché et la vulgarité d'un matérialisme philistin, tout ceci contribuant à la dégradation d'un environnement urbain dont Morris souligne la laideur ${ }^{6}$. L'environnement naturel ne déroge pas à la règle, Morris décrit une nature exploitée et bafouée, soumise aux diktats du monde des manufactures, dont le fleuve souillé par les déchets de l'Angleterre industrielle est la meilleure illustration.

9 Le motif de la dégénérescence s'applique également aux corps, et aux vêtements qui les parent. Comparée aux formes épurées et aux riches étoffes qui reflètent et mettent en 
valeur la beauté des corps de ces magnifiques spécimens de l'espèce humaine qui peuplent le pays de Nulle Part, la mode victorienne est tournée en ridicule, qui transforme les femmes en fauteuils. La plume de Morris « croque » les corps minaudant des bourgeoises, le physique ingrat des oisifs (lire «les intermédiaires») et les corps malingres et douloureux des pauvres. Si la chair est triste dans l'Angleterre victorienne, le corps social est également frappé de dégénérescence. Les relations sociales s'établissent exclusivement sur le mode complexe et conflictuel des relations de pouvoir. Morris déplore la perversion des relations entre les sexes dont l'institution du mariage est l'exemple le plus abouti : les femmes y sont soumises et impuissantes, le couple y est condamné au respect de conventions sociales étriquées; les relations humaines sont perverties par l'établissement d'une société de classes comme en témoigne le regard servile du sous-homme que croise William Guest à la fin du récit. Enfin, News from Nowhere propose en creux une dénonciation de relations de production dégradées, organisées exclusivement sur le mode de l'exploitation et de la coercition.

10 Autant de symptômes de la dégénérescence de la société victorienne et du caractère mortifère de l'ordre bourgeois. Au-delà du constat, le diagnostic suggéré par Morris s'apparente à la critique "romantique » du capitalisme industriel et de l'utilitarisme : le lecteur perçoit notamment des échos de la théorie du cash nexus élaborée par Carlyle ${ }^{7}$, la dénonciation, dans la tradition de la critique radicale, d'un système à la fois inique et inepte qui place le monde du capital et le monde du travail dans une relation exclusivement antagoniste, et qui accorde la priorité au profit et à la rentabilité soumettant ainsi le système productif aux impératifs de la commercialisation. Enfin, on trouve au cœur de la critique morrissienne la notion centrale d'aliénation déclinée en deux modalités: l'aliénation technologique tout d'abord qui dénonce l'impact de la mécanisation sur l'activité productive, l'ouvrier étant à la fois placé en concurrence avec la machine et soumis par le biais de la spécialisation du travail imposée par le machinisme à l'obligation de s'acquitter de tâches mécaniques et répétitives ; en second lieu, l'aliénation par le marché ou market alienation qui dénonce une autre forme de dépossession, l'absence de contrôle du producteur sur le résultat de son travail. L'importance accordée à la notion d'aliénation atteste de la proximité de la pensée morrissienne avec la théorie marxiste, toutefois on trouve également dans News from Nowhere des échos de la notion durkheimienne d'anomie dans l'évocation en creux du processus de désintégration à l'œuvre dans la société victorienne. Morris y dénonce à la fois l'absence de lien social et l'isolement de l'ouvrier dans son activité productive ${ }^{8}$.

11 News from Nowhere permet de mesurer l'évolution du parcours individuel de William Morris, du rejet des codes esthétiques de l'Angleterre victorienne à la prise de conscience du caractère fallacieux de l'idée selon laquelle l'art et l'artiste peuvent se développer en marge de la société; l'entrée en politique de Morris procède de la prise de conscience de l'impact du capitalisme sur l'activité artistique et de l'importance des codes culturels dominants qui président à la fois à l'organisation de la société ainsi qu'à la structuration de toute activité productive, y compris artistique. Nouvelles de Nulle Part signale également une autre transition : certes, Morris s'inscrit dans la tradition de la critique romantique où s'exprime la nostalgie d'un ordre antérieur témoignant de la force d'attraction du modèle médiéval; par certains aspects, sa dénonciation des mutations socio-économiques qui ont fait voler en éclat le système pré-capitaliste en introduisant une dynamique délétère rejoint les préoccupations de certains tories. Mais 
William Morris n'est pas Walter Scott et le «médiévisme » de News from Nowhere est au service d'un tout autre projet.

\section{Les chemins qui mènent à Nulle Part}

Comme le rappelle Clive Wilmer dans son introduction à l'édition Penguin de News from Nowhere, la structure de l'œuvre est d'une grande simplicité : deux voyages, l'un terrestre, l'autre fluvial, encadrent le récit des événements qui ont produit le bouleversement dont William Guest découvre les effets. Au cœur du texte se trouvent donc deux chapitres ${ }^{10}$ à vocation heuristique qui conduisent le lecteur à réviser sa perception du monde de Nulle Part, tout en explicitant le sous titre 'An epoch of rest': il ne s'agit plus uniquement d'une chimère, simple produit d'une imagination fertile et d'une rêverie un peu vaine, mais aussi et peut-être surtout de proposer une incarnation des objectifs de la cause révolutionnaire tout en affirmant la nécessité de la révolution socialiste. Morris passe ainsi du rêve à la vision : il s'agit pour lui de donner corps au désir des socialistes et de démontrer la nécessité de l'action. Enfin, le lecteur peut alors préciser la nature du lien qui unit les deux mondes de l'utopie morrissienne tout en réintroduisant du sens dans l'apparente confusion chronologique d'un texte où se superposent passé, présent et avenir ${ }^{11}$.

13 Morris a indéniablement accordé une grande attention au récit de l'épisode révolutionnaire qui ouvre l'ère du socialisme. Le texte témoigne du soin apporté à la rédaction du passage : le souci de réalisme dans le détail de la confrontation le dispute au souci de vraisemblance dans la description de l'enchaînement des événements. Le récit dans lequel on trouve des échos d'autres épisodes révolutionnaires ou insurrectionnels antérieurs tels que la Révolution Française (Committee of Public Safety) ou encore les affrontements entre les partisans du changement et les forces de la réaction qui jalonnent le XIX ${ }^{\mathrm{e}}$ siècle, tels Peterloo, l'épisode éphémère de la Commune de Paris, ou bien encore Bloody Sunday, où le bras armé de l'ordre bourgeois a réprimé dans le sang des manifestations pacifiques.

L'évocation du fait révolutionnaire place Morris dans le camp des partisans d'une rupture totale avec le capitalisme industriel et le libéralisme économique. Le message transmis par l'auteur de News from Nowhere insiste sur la nécessité d'une métamorphose, d'un changement radical, qui seuls peuvent garantir l'avènement d'un ordre nouveau. Par ailleurs, Nouvelles de Nulle Part s'inscrit alors dans le schéma utopique de la transformation volontaire ou willed transformation ${ }^{12}$ qui permet à l'auteur de prendre ses distances avec tout évolutionnisme historique.

Malgré toute la gravité du propos, Nouvelles de Nulle Part n'est pas une œuvre dénuée d'ironie, voire d'autodérision et Morris s'y montre parfois sensible aux vertus de la distanciation humoristique. Le récit s'ouvre in medias res au beau milieu de ce que le lecteur ne peut manquer d'identifier comme une réunion d'un groupuscule socialiste ; l'auteur souligne avec malice les divergences qui opposent les différents membres du groupe: 'There were six persons present, and consequently six sections of the party were represented, four of which had strong but divergent Anarchist opinions. ${ }^{13}$ L'ironie est mordante mais le trait est précis et ces quelques lignes en disent long sur la tendance à la fragmentation qui caractérise le mouvement socialiste à ses débuts. William Morris avait une expérience directe de ces réunions et une conscience aiguë des effets délétères des querelles idéologiques mais aussi du choc des ambitions personnelles. Il 
avait également été à l'origine d'une scission au sein de la Social Democratic Federation qu'il avait quittée en compagnie de Bax, Aveling et Eléonore Marx, pour fonder la Socialist League ${ }^{14}$. Les tensions au sein de la famille socialiste ne manquaient donc pas, comme Morris l'admet volontiers.

Il semble néanmoins que l'opposition la plus significative qui permette de saisir par contraste la spécificité de l'idéal socialiste morrissien reste celle qui a opposé l'auteur de News from Nowhere à la pensée fabienne. L'option révolutionnaire défendue par William Morris le distingue de cet autre courant de pensée qui a participé à l'élaboration d'un discours socialiste dès la fin du XIX siècle. Le nom choisi par les fondateurs de la Société fabienne par la référence à l'antique figure de Fabius qui se prononça en faveur de la modération et du changement progressif ${ }^{15}$ affiche ainsi le choix d'un gradualisme justifié par le positivisme et une confiance inébranlable en une forme de déterminisme darwinien. Au caractère inéluctable du gradualisme fabien, ${ }^{16}$ William Morris oppose l'inévitabilité de la révolution. Le Morris de News from Nowhere se démarque également de la pensée fabienne dans la critique qu'il propose du capitalisme industriel et, partant, dans la substance même de l'alternative socialiste qu'il propose.

17 Si la critique du capitalisme que proposent les Fabiens n'est pas dénuée de compassion pour la misère du prolétariat et donc d'une composante morale, celle-ci se cristallise néanmoins essentiellement autour d'une dénonciation des imperfections et des dysfonctionnements inhérents au système. La pauvreté notamment, que Beatrice Webb tente d'identifier et de quantifier en collaborant à son évaluation chiffrée avec Charles Booth, est perçue comme la conséquence d'une distribution inefficace des revenus et des richesses. Au cœur de la pensée fabienne se trouve une dénonciation des monopoles qui s'inscrit dans la lignée des travaux de Ricardo et notamment de sa theory of rent. La solution aux yeux des fabiens réside dans l'appropriation collective de l'outil de production par l'État, garant de la redistribution des richesses et de la mise en œuvre d'outils scientifiques de gestion de l'économie et notamment du secteur productif. Il en découle que la stratégie privilégiée par la Société fabienne est une stratégie réformiste, visant à réparer les injustices et à améliorer les performances du système capitaliste ; le socialisme peut alors être perçu comme un stade supérieur de développement et non comme un nouveau modèle d'organisation sociale régi par des règles économiques et une éthique radicalement différentes.

Le collectivisme réformiste qui caractérise la pensée fabienne est contesté par l'auteur de News from Nowhere au prétexte que le simple transfert d'autorité à l'État ne pourra que remplacer un monopole privé par un monopole public; Morris n'y voit pas la véritable diffusion du pouvoir qui caractérise à ses yeux le socialisme. Par ailleurs, le réformisme n'altère en rien la logique même du système capitaliste, au mieux les réformes ne peuvent que permettre à une fraction de la classe ouvrière d'améliorer ses conditions de vie et de travail et d'accéder aux privilèges dont jouissent les classes moyennes, aux dépens des plus démunis ${ }^{17}$. La logique d'exploitation et d'aliénation qui caractérise le capitalisme marchand n'est pas remise en cause mais plutôt légitimée ${ }^{18}$. William Morris a su à l'occasion se montrer très sévère à l'égard des partisans de l'option collectiviste et réformiste, accusés de promouvoir un socialisme « au rabais » et parfois même de faire le jeu des adversaires de la classe ouvrière ${ }^{19}$. Il rejette de toutes ses forces l'économisme fabien et son approche exclusivement comptable, unidimensionnelle et donc réductrice de la dignité humaine pour y substituer une 
vision qu'il souhaite plus dense, plus complexe, plus stimulante mais aussi plus conforme à la vérité du genre humain.

\section{La Nouvelle Jérusalem}

19 C'est la vocation d'une utopie que de s'attacher à la création d'un ordre nouveau et William Morris fait effectivement œuvre de démiurge dans News from Nowhere, tout en plaçant le concept de création au cœur de sa réflexion. Nulle Part est tout d'abord un monde "désinstitutionnalisé » qui se caractérise par l'intériorisation des règles qui régissent le corps social. Les codes qui encadrent l'activité humaine ont été si bien intégrés par la population que l'existence d'institutions censées représenter la loi ou bien la faire respecter en est devenue superflue tant la morale individuelle et collective relève de l'habitude. On sait que William Morris n'est pas resté insensible aux discours des anarchistes comme Kropotkine, toutefois Nulle Part n'est pas le rêve d'un anarchiste : il n'y est jamais question de se dispenser des lois et de rejeter toute règle. De même, les accusations d'angélisme, si elles ne sont pas totalement dénuées de fondement, négligent cependant la spécificité de la conception morrissienne de l'ordre nouveau.

Nulle Part n'est pas un nouvel Éden qui ne connaît pas (ou plus) le mal. La société y est bel et bien confrontée aux pulsions criminelles ou "négatives"; tout l'intérêt de l'utopie morrissienne est de présenter un nouveau mode d'organisation sociale où la gestion de l'acte criminel par la communauté a été résolue sans qu'il fût nécessaire d'avoir recours à la force, à la contrainte ou à la violence. Il est alors possible de percevoir News from Nowhere comme une forme d'inversion du schéma de la théorie politique classique qui décrit le passage de l'état de nature à l'état social par le biais d'un transfert d'autorité à une institution qui devient l'incarnation de la volonté collective et le garant du bien commun. Néanmoins, il serait tout à fait erroné d'y voir une sorte de retour à l'état de nature; au contraire, Morris décrit dans Nulle Part le stade ultime de développement de toute société organisée qui transcende l'autorité de l'État ${ }^{20}$ en assurant sa diffusion au sein de la communauté.

Le processus de «désinstitutionnalisation » qui procède de l'épisode révolutionnaire, cet effacement progressif des institutions (withering away of the State), ne s'applique pas à l'État uniquement dans sa dimension juridique et répressive. C'est également en tant qu'incarnation de l'idéal collectif que l'État est voué à la disparition. Morris y substitue une autre modalité de la collectivité par le biais de l'évocation de ces communautés "immédiates ", unités de base de l'organisation sociale de Nulle Part et expression à la fois la plus simple et la plus aboutie de la solidarité et de la fraternité qui unissent les habitants de Nulle Part. News from Nowhere est dominé par le motif de la réconciliation des contraires. Les exemples sont nombreux et de façon significative le texte de Morris célèbre la réunion de l'individuel et du collectif. Si les personnages de Nulle Part sont avant tout des archétypes dont on peut regretter le manque de densité et de profondeur psychologique, Morris veille néanmoins à les différencier. Le propos est démonstratif, loin de s'annuler, l'individuel et le collectif se renforcent mutuellement : l'organisation communautaire favorise la diversité et la solidarité s'établit entre des individus conscients de leur valeur intrinsèque, dont la singularité est soulignée de façon systématique. Cette complémentarité s'exprime avant tout dans et par le travail. 
Nouvelles de Nulle Part n'est pas une utopie de la libre consommation, tout comme Nulle Part n'est pas un pays de cocagne. Si les hommes y goûtent les fruits qu'une nature bienveillante met à leur disposition, la consommation s'y assortit systématiquement d'une transformation. Le travail est du reste l'élément central de l'utopie morrissienne, ainsi que le principe structurant de la nouvelle société de Nulle Part. La population y est regroupée en petites communautés de producteurs, autonomes et solidaires, organisées sur le modèle coopératif. L'immense majorité des habitants est occupée à des tâches productives, au sens large du terme, sans qu'aucune hiérarchisation ne vienne troubler l'harmonie ambiante ${ }^{21}$. Le travail y est organisé sur les bases d'une théorie de la valeur « revisitée " par William Morris. La valeur, telle qu'elle est mesurée par les habitants de Nulle Part, n'est pas déterminée par les lois du marché : la monnaie n'a du reste plus cours, les quelques pièces noircies enfouies dans la poche de William Guest ne lui seront d'aucune utilité durant son périple et susciteront la surprise et l'incompréhension de ses hôtes. De même, le travail n'est plus une activité salariée ; la valeur réelle du travail ou d'un bien de consommation quelconque se mesure à présent en fonction de son utilité à la fois pour l'individu et la communauté : la production répond à une évaluation des besoins en termes quantitatifs et qualitatifs et seuls les travaux indispensables à la vie de la communauté sont effectués. Le critère d'utilité garantit la valorisation des tâches traditionnellement considérées comme subalternes, travaux ménagers ou entretien des voies de communication, tout comme le principe de la nécessaire adéquation de la production et des besoins impose le modèle de petites unités de production organisées sur une base artisanale.

L'économie de Nulle Part ne génère donc pas d'excédent et ne laisse ainsi aucune place à la commercialisation et au profit. Les conséquences en sont à la fois multiples et variées: l'absence de commercialisation dispense les habitants de Nulle Part de l'obligation d'organiser leur production dans un contexte concurrentiel qui justifie, pour assurer la conquête de nouveaux marchés, la dégradation des conditions de travail et de rémunération des ouvriers mais aussi peut-être la mise en œuvre d'une politique étrangère agressive visant à garantir la suprématie sur le marché international et aussi l'assurance d'une rente substantielle pour les profits investis à l'étranger ${ }^{22}$. L'absence d'impératifs liés à la commercialisation d'une production de masse permet également de libérer l'ouvrier de toute une série de contraintes, spécialisation et division du travail ${ }^{23}$, augmentation des cadences et du temps de travail, qui sont génératrices d'aliénation et de déshumanisation.

La valeur telle qu'elle est définie dans Nouvelles de Nulle Part est également fonction du travail nécessaire à la production. William Morris se concentre essentiellement sur la valeur réelle de l'objet, établie en fonction de la quantité et de la qualité du travail investi dans sa fabrication. Cette définition est à l'évidence incompatible avec les impératifs de la production de masse et l'on retrouve ici les préoccupations du cofondateur du mouvement Arts and Crafts. Le travail est assimilé à un acte de création, la plus haute expression de l'humanité de son créateur, la valeur de l'objet procédant alors à la fois de la maîtrise d'une technique et de la créativité de l'artiste-artisan ${ }^{24}$. Cette conception de la valeur témoigne d'une idée élargie des «besoins » de l'individu et de la communauté tout entière qui ne sauraient être évalués en termes exclusivement matériels et économiques, au détriment de toute dimension sociale, éthique ou esthétique. C'est dans cette perspective que l'expression joyful labour ${ }^{25}$, prend tout son sens, par opposition à useless toil ${ }^{26}$. 


\section{L'héritage morrissien} antagonismes générés par le capitalisme marchand sont transcendés pour laisser place à une société fraternelle et solidaire. La vision morrissienne, pour séduisante qu'elle fût, n'a toutefois pas été épargnée par la critique. À l'évidence, Nouvelles de Nulle Part n'est pas un traité d'économie politique, et comparée aux travaux des théoriciens socialistes l'œuvre souffre d'un déficit «scientifique». William Morris convenait du reste volontiers de ses limites en matière de théorie économique, comme le démontre l'épisode célèbre qui voit l'auteur de News from Nowhere déclarer lors d'un meeting à Glasgow, non sans une pointe de provocation :

Truth to say, my friends, I have tried to understand Marx's theory, but political economy is not in my line, and much of it appears to me to be dreary rubbish. But I am, I hope, a Socialist none the less. It is enough political economy for me to know that the idle class is rich and the working class is poor, and that the rich are rich because they rob the poor. ${ }^{27}$

Enfin, l'idéalisation de la société médiévale, pré-capitaliste et préindustrielle avec un Morris tournant résolument le dos aux progrès de la technique souligne encore davantage le décalage de la pensée morrissienne avec le positivisme qui domine la réflexion de ses contemporains. News from Nowhere a néanmoins reçu un accueil chaleureux dans les cercles socialistes et figure en bon rang parmi les œuvres préférées d'une large proportion des membres du nouveau parti travailliste ${ }^{28}$. Parmi ceux qui voient dans News from Nowhere l'œuvre la mieux à même d'exprimer l'idéal socialiste se trouvent les Guild Socialists, ce mouvement à l'existence assez éphémère, qui connut son apogée au terme de la Première guerre mondiale ${ }^{29}$ pour tomber dans un oubli relatif de façon assez soudaine dès la fin des années 1920. Les Guild Socialists se définissent en premier lieu comme un groupe de réflexion et un groupe de pression. Leurs publications ont pour vocation d'alimenter le débat d'idées tout en faisant contrepoids aux thèses collectivistes et réformistes qui ont le vent en poupe au sein de la Société fabienne. Dans un second temps, et plus précisément lorsque l'échec de cette politique d'entrisme devint patent, ils eurent recours à une stratégie différente. Avec la création de la National Guilds League en 1915, le mouvement s'émancipe de la tutelle de la Société fabienne et change ses modalités d'action : il s'agit alors d'ancrer le mouvement dans la réalité en privilégiant l'action concrète sur le terrain.

A.J. Penty, qui publie The Restoration of the Gild System en 1906, fait traditionnellement figure de précurseur du Guild Socialism. Les pistes de réflexion évoquées dans cette œuvre de pionnier sont reprises et prolongées à partir de 1907 dans les colonnes de The New Age d'Alfred Orage, qui devient rapidement l'un des organes officiels des Guild Socialists. Pour l'essentiel, le courant Guild Socialist fut représenté et amené à maturité par G.D.H. Cole et S.G. Hobson ${ }^{30}$, auteurs prolifiques et personnalités influentes et respectées au sein du mouvement travailliste. Le propos évolue donc sous la plume de différents auteurs qui tour à tour se réclament du Guild Socialism tout en lui donnant des inflexions particulières ${ }^{31}$. Ce n'est nullement le lieu d'entrer ici dans le détail des prises de position individuelles et divergences qui ne manquèrent pas d'apparaître mais il s'agit plutôt d'identifier le socle commun sur lequel reposent les travaux des Guild Socialists. À l'évidence, l'héritage morrissien que revendiquent ces auteurs constitue l'une des polarités fortes de leur réflexion. La dette des Guild Socialists envers William

Revue Française de Civilisation Britannique, XIII-1 | 2004 
Morris se manifeste tout d'abord dans leur critique du capitalisme industriel et marchand. Les emprunts à l'auteur de News from Nowhere sont légion et l'on perçoit à travers les références évidentes aux écrits du sage de Kelmscott une adhésion aux thèses développées par la critique romantique qui inspira si profondément Morris ${ }^{32}$.

C'est toutefois dans la conception d'un ordre nouveau ainsi que dans la définition des buts ultimes du socialisme et des moyens d'y parvenir que la filiation s'impose avec une évidence incontestable. Aux yeux des Guild Socialists, le socialisme a pour mission de libérer l'individu de l'asservissement que lui impose un système de production organisé sur des bases capitalistes ainsi que son corollaire, le salariat ou wage-slavery. Certes, le concept de libération n'est pas absent, loin s'en faut, de la réflexion d'autres théoriciens du socialisme, mais l'idéal de liberté est non seulement au cœur de la réflexion menée par les Guild Socialists, mais encore il procède d'une série d'exigences qui font la spécificité du Guild Socialism tout en révélant le caractère central de l'héritage morrissien.

29 La réflexion des Guild Socialists se distingue en premier lieu par la primauté accordée à la question de l'organisation du secteur productif. Leurs écrits envisagent un système de production décentralisé, organisé sur le modèle des guildes du Moyen Âge, ces corporations ou communautés de producteurs dont l'une des principales caractéristiques est qu'elles jouissent d'une réelle autonomie. Cette réorganisation est conçue comme une sorte d'antidote aux tendances centralisatrices du collectivisme fabien; elle se justifie également par la nécessité de soustraire la production aux exigences de la commercialisation et le producteur aux exigences du salariat. Aux yeux des Guild Socialists, la problématique qui consiste à opposer secteur public et secteur privé est une simplification à outrance qui ne permet pas de saisir les véritables enjeux de la révolution socialiste et se concentre sur ces problèmes annexes que sont les questions de redistribution sans modifier en profondeur le statut de l'ouvrier; ce dernier demeure le simple rouage d'un mécanisme sur lequel il n'a aucune prise et reste soumis à des impératifs de production dictés par le marché. Seule une organisation de la production sur des bases entièrement repensées permet d'investir l'activité productive d'une signification allant au-delà de la simple satisfaction d'une demande. Le système des guildes permet aux producteurs d'organiser leur activité à partir de critères non marchands, en intégrant des paramètres qui confèrent au travail de l'artisan-producteur une valeur morale, esthétique et politique.

Les Guild Socialists militent également en faveur d'un nouvel équilibre des forces entre le secteur productif, le secteur marchand et l'État. Le système qu'ils imaginent suppose une décentralisation de l'activité productive et un transfert de pouvoir. Il s'agit de restituer aux producteurs un pouvoir de décision qui leur a été confisqué ; les guildes y définissent les modalités de la production ainsi que ses objectifs. S'il existe bien des divergences entre les partisans d'une autonomie complète des associations de producteur et ceux qui comme G.D.H. Cole sont convaincus de la nécessité de coopérer avec l'État, elles ne remettent pas en cause le principe essentiel de la décentralisation du pouvoir. La revalorisation du rôle et du statut du producteur passe également par une réorganisation de l'entreprise qui applique le même principe de diffusion du pouvoir. Les Guild Socialists se prononcent en faveur de l'instauration d'une véritable démocratie participative au sein de l'entreprise imposant ainsi les principes de la consultation régulière et de l'association des ouvriers à la prise de décision. En cela le Guild Socialism fait écho aux préoccupations des partisans d'un système autogéré. 
31 Par ailleurs, les Guild Socialists expriment une profonde insatisfaction à l'égard du fonctionnement de la vie démocratique en Grande-Bretagne et dénoncent une conception trop étriquée de la démocratie lorsque celle-ci ne s'exerce que par le biais du suffrage. Ils font également preuve d'un réel scepticisme quant à la possibilité de promouvoir le changement par la voie parlementaire. La mise en place du suffrage universel en 1918 ne satisfait pas leurs exigences en matière de démocratie et ne répond que très partiellement à leurs attentes. Dans la mesure où le système politique n'est que le reflet d'un rapport de forces défavorable à la classe ouvrière, l'avènement du socialisme, contrairement à ce qu'affirment les fabiens, ne peut pas procéder d'une stratégie parlementaire. Dans des textes qui font écho à l'irrévérence de William Morris à l'endroit du pouvoir et de la représentation nationale ${ }^{33}$, les Guild Socialists se déclarent favorables à une autre forme d'action, indépendante de l'action politique, en appelant à la mobilisation de la classe ouvrière.

32 Enfin, parmi les désaccords qui opposent les Guild Socialists aux fabiens, tout en faisant écho à la réflexion de William Morris, il faut citer le rejet du collectivisme qui néglige, à leurs yeux, ces groupements intermédiaires qui font la richesse du tissu social et qui sont autant d'expressions de la diversité des modes de socialisation. On trouve donc dans les écrits des Guild Socialists des échos de la pensée pluraliste, perceptible dans les écrits de Morris et prolongée notamment par Harold Laski, ainsi que le souci d'éviter toute dérive étatique en compensant les pouvoirs excessifs de l'État grâce à la revitalisation de ces groupements intermédiaires qui fonctionnent comme autant de contre-pouvoirs, les guildes certes, mais aussi la famille, les associations, les municipalités et les Églises ${ }^{34}$.

33 En soi, le Guild Socialism est un témoignage de la vitalité de la pensée morrissienne. La voix de l'auteur de News from Nowhere a su trouver un écho auprès de penseurs exigeants et influents tels G.D.H. Cole ou S.G. Hobson; ces derniers ont assuré la participation posthume de William Morris au grand débat d'idées qui a conduit à l'émergence du travaillisme en Grande-Bretagne. Les Guild Socialists ont également fait la démonstration que l'œuvre de Morris, et plus particulièrement News from Nowhere, remplit les critères de l'utopie scientifique pour reprendre la terminologie employée par F. Engels.

Nouvelles de Nulle Part n'appartient pas au genre de la littérature d'évasion mais propose bel et bien une vision du sens de l'histoire ainsi qu'un programme d'action. Quelles que soient leurs divergences, les Guild Socialists ont tenté de traduire dans la réalité concrète la vision morrissienne de la société socialiste. Ils ont engagé une restructuration complète de l'économie sur des bases coopératives et défini des modalités d'action pour mettre fin au système capitaliste. Des contacts ont été établis avec les syndicats pour convertir le monde du travail au Guild Socialism et transformer ces organisations en instruments du changement. Les modalités du transfert de pouvoir aux guildes ont été définies avec précision, de la ligne dure favorable à un rapprochement avec le socialisme révolutionnaire à l'option plus modérée d'une expropriation progressive connue sous le nom de encroaching control ${ }^{35}$. Enfin, il faut porter à l'actif des Guild Socialists la création de communautés coopératives telles la National Guild of Builders.

Certes, le mouvement fut éphémère et il est possible de percevoir le Guild Socialism comme une simple parenthèse dans l'histoire du socialisme britannique. Sa dimension conjoncturelle milite en faveur de cette interprétation. Après des débuts hésitants, les Guild Socialists ont bénéficié de circonstances propices au développement ainsi qu'à la 
diffusion de leurs idées. Les porte-parole du mouvement ont écrit dans un environnement social favorable qui les a assurés de rencontrer un large écho auprès d'un mouvement syndical en plein essor : le nombre de syndiqués augmente de façon très nette de 4,2 à 8,1 millions entre 1913 et 1919, le militantisme ouvrier, en phase ascendante dès 1910-1912, atteste de la radicalisation du mouvement ${ }^{36}$ et les grandes centrales syndicales manifestent leur volonté d'en découdre en organisant la coopération au sein de la Triple Alliance fondée en 1914. Comparée à la montée en puissance d'un mouvement de contestation qui s'organise sur des bases industrielles, l'action politique faisait pâle figure: le jugement Osborne de 1909, qui mit temporairement fin au financement du parti travailliste par les syndicats, fut la source d'un désenchantement confirmé par la relative impuissance des députés travaillistes au Parlement. Les Guild Socialists ont été portés par des vents ascendants: grâce au rapprochement avec le socialisme révolutionnaire, ils rencontrent les préoccupations d'autres partisans de l'autogestion comme la CGT en France ou le leader du Socialist Labour Party américain, Daniel de Leon ${ }^{37}$. De même, le déclin du mouvement peut s'expliquer par l'apparition d'un nouvel environnement social et politique dont les Guild Socialists firent les frais.

Quel bilan faut-il alors dresser de l'expérience du Guild Socialism en Grande-Bretagne ? À l'évidence, les Guild Socialists font figure de grands vaincus de l'histoire; ils se sont montrés incapables de s'opposer durablement à la progression des thèses collectivistes et réformistes et ne purent donc relever le défi lancé par les ténors de la Société fabienne ; la disparition soudaine d'un mouvement rejeté aux marges de l'histoire du socialisme britannique atteste de son incompatibilité avec les deux modalités dominantes du socialisme au $\mathrm{XX}^{\mathrm{e}}$ siècle, le travaillisme et le communisme. Toutefois, si le mouvement fut éphémère, la permanence du questionnement initié par William Morris et prolongé par le Guild Socialism s'impose comme une évidence. Les préoccupations libertaires de l'auteur de News from Nowhere réapparaissent régulièrement sous la plume de ces auteurs qui ont tenté de réduire la fracture entre socialisme et liberté, en même temps qu'elles constituent l'une des idiosyncrasies de la pensée socialiste britannique.

\section{NOTES}

1. E.P. THOMPSON, William Morris : Romantic to Revolutionary, London: Lawrence \& Wishart, 1955 (London: Merlin Press, 1997).

2. Voir la postface de 1976 où Thompson propose une lecture critique du texte de Meier, La pensée utopique de William Morris, Paris : Éditions sociales, 1972.

3. Ayant conscience du caractère réducteur d'une lecture de News from Nowhere qui serait dominée par la question exclusive de sa conformité au dogme marxiste, E.P. Thompson propose de déplacer la problématique en suggérant que les tensions qui subsistent entre le texte morrissien et le marxisme "officiel » s'expliquent par la rigidité doctrinale et l'ossification prématurée de la théorie marxiste à la fin du XIX ${ }^{\mathrm{e}}$ siècle. 
4. L'expression est empruntée à Franck LESSAY dans sa présentation des diverses modalités de l'utopie rattachée à une étude de l'Atlantide de Francis Bacon, «De l'histoire rêvée à l'histoire réelle : sur La Nouvelle Atlantide de Francis Bacon », Revue Française de Civilisation Britannique, Hors Série $n^{\circ} 1$, mars 2001 , pp. 29-41. Pour une typologie de l'utopie on se reportera également avec profit à l'article de Raymond WILLIAMS, 'Utopia and Science Fiction', Science Fiction Studies, vol. 5, $n^{\circ}$ 3, November 1978.

5. Raymond WILLIAMS, ibid.

6. A la date où écrit Morris, le mouvement des utopies urbaines est en plein essor. On accorde une nouvelle importance à l'aménagement des villes et les cités modèles imaginées quelques années plus tard, comme les cités jardins d'Ebenezer Howard, sont autant d'utopies sociales. On lira à ce propos l'ouvrage de Gordon E. CHERRY, Cities and Plans, London: Edward Arnold, 1988.

7. L'expression est employée par l'auteur de Past and Present pour décrire des relations de production où la responsabilité de l'employeur envers ses ouvriers se limite au versement d'un salaire dont le montant est fixé par la loi de l'offre et de la demande. Le travail est alors un simple bien de consommation (commodity) qui s'échange sur le marché comme tout autre bien de consommation.

8. Pour davantage de précisions on peut se reporter à l'ouvrage d'Anthony GIDDENS, Capitalism and modern social Theory: An Analysis of the Writings of Marx, Durkheim and Max Weber, Cambridge: C. U. P., 1971, et notamment aux pages 228-229.

9. Morris écrivait: 'Believe me, it will not be possible for a small knot of cultivated people to keep alive an interest in the art and records of the past amidst the present conditions of a sordid and heart-breaking struggle for existence for the many, and a languid sauntering through life for the few.' In May MORRIS (ed.), William Morris : Artist, Writer, Socialist, Oxford: Blackwell, 1936, vol. 1, p. 145. Cité dans E.P. THOMPSON, William Morris : Romantic to Revolutionary, p. 241.

10. Chapitre XVII, 'How the Change Came' et chapitre XVIII, 'The Beginning of the New Life'.

11. La question du traitement du temps et de l'histoire dans News from Nowhere mériterait que l'on s'y arrête plus longuement. Contentons-nous ici de souligner l'existence de parallèles entre le texte de Morris et le matérialisme historique marxiste qui désigne la lutte des classes comme moteur de l'histoire et qui définit l'avenir en termes essentiellement qualitatifs comme le développement d'un potentiel humain dont les traces parfois virtuelles sont perceptibles dans des modèles antérieurs d'organisation économique et sociale.

12. Raymond WILLIAMS, 'Utopia and Science Fiction'.

13. William MORRIS, News from Nowhere and Other Writings, London: Penguin, 1998 (1891), p. 43.

14. Sur tous ces points, notamment l'expérience de William Morris en tant que militant et la fondation de la Socialist League, voir E.P. THOMPSON, William Morris : Romantic to Revolutionary.

15. Vincent Geoghegan cite une partie de la profession de foi des premiers fabiens : 'For the right moment you must wait, as Fabius did most patiently, when warring against Hannibal, though many censured his delays', 'Socialism' in R. ECCLESHALL, V. GEOGHEGAN, R. JAY \& R. WILFORD (eds.), Political Ideologies : An Introduction, London: Unwin Hyman, 1984, p. 134.

16. 'The inevitability of gradualness', l'expression est de Sidney Webb.

17. On peut lire dans la correspondance de Morris: 'Much as I want to see workmen escape their slavish position, I don't at all want to see a few individuals more creep out of their class, into the middle class; this will only make the poor poorer still', The Letters of William Morris to his Family and Friends, Philip Henderson (ed.), London: Longmans Green \& $C^{\circ}, 1950$, pp. 197-199, cité par E.P. THOMPSON, William Morris : Romantic to Revolutionary, p. 320.

18. William Morris écrit dans Commonweal : 'I should like our friend to understand whither the whole system of palliation tends - namely, to the creation of a new middle class to act as a buffer between the proletariat and their direct and obvious masters; the only hope of the bourgeois for retarding the advance of Socialism lies in this device', 'Socialism and Politics', Commonweal, vol. 1, n6, Supplement (July 1885), p. 61. Reproduit dans William MORRIS, Political Writings: Contributions to Justice and 
Commonweal 1883-1890. Edited and Introduced by Nicholas Salmon. Bristol: Thoemmes Press, 1994, pp. 98-100 (p. 99).

19. Morris n'a pas de mots assez durs pour condamner l'option réformiste privilégiée par la Société fabienne. Il écrit notamment à propos de Sidney Webb : 'What is the real gate which will pull up these soft Socialists, who so long as they are allowed to steal the goose will not object to give the giblets to the poor.' 'Notes on News', Commonweal, vol.5, n¹94 (28 September 1889), p. 305. Cité dans E.P. Thompson, William Morris: Romantic to Revolutionary, p. 682. Notons néanmoins que William Morris saura ultérieurement tempérer son animosité à l'égard des Fabiens et nuancer ses propos en convenant de ce que le collectivisme réformiste peut constituer une étape sur la voie du socialisme. C'est du reste le cas dans News from Nowhere, comme en attestent les pages 137-138.

20. À l'évidence, William Morris adhère à la théorie marxiste qui voit dans l'état la manifestation institutionnelle du pouvoir des classes dominantes.

21. Seul le travail "intellectuel», non productif, y fait l'objet de quelques railleries dans un renversement ironique de la hiérarchie traditionnelle.

22. L'anti-impérialisme de William Morris est une des raisons qui l'ont détourné du Parti libéral de Gladstone. On peut percevoir dans News from Nowhere des analyses qui suggèrent une proximité de vue avec l'un des premiers théoriciens de l'anti-impérialisme, J.A. Hobson.

23. Dans le pays de Nulle Part, le travail est mis en commun et si Morris tient compte des compétences individuelles il n'en affirme pas moins la nécessité de la diversification des tâches.

24. L'un des personnages de News from Nowhere déclare : 'We have learned the trick of handicraft, and added the utmost refinement of workmanship to the freedom of fancy and imagination.' News from Nowhere, p. 201.

25. La formule est de RUSKIN, 'Art is the expression of man's joy in labour'.

26. William Morris, 'Useful Work versus useless Toil' (1884). Reproduit dans Clive WILMER (ed.), News from Nowhere and Other Writings, Harmondsworth: Penguin, 1993 (1998), pp. 283-306.

27. Ces propos sont rapportés par John Bruce GLASIER dans William Morris and the Early Days of the Socialist Movement, 1921, pp. 31-32. Cité par E.P. THOMPSON, William Morris: Romantic to Revolutionary, p. 356.

28. Noel Thompson livre le résultat d'un sondage effectué auprès des 29 députés travaillistes nouvellement élus en 1906, qui place News from Nowhere en troisième position au palmarès des œuvres les plus influentes après la Bible et The Pilgrim's Progress de John Bunyan. Noel THOMPSON, Political Economy and the Labour Party, London: University College London Press, 1996, p. 11.

29. Les Guild Socialists ne constituèrent jamais un mouvement de masse : à son apogée en 1921 la National Guilds League comptait environ 500 adhérents. Voir W.H. GREENLEAF, The British Political Tradition, Vol. 2 : The Ideological Heritage, London: Methuen, 1983, p. 427.

30. G.D.H. Cole publie deux ouvrages majeurs, Self-government in industry, London: Bell, 1917 et Guild Socialism restated, London: Parsons, 1920. L'œuvre la plus significative de S.G. Hobson est sans conteste National Guilds : An Inquiry into the Wage System and the Way out, London: Bell, 1914.

31. Le mouvement ne fut jamais monolithique ; parmi les principales lignes de fracture citons, la question controversée du progrès technologique et de la mécanisation, et les divergences concernant le rôle de l'État qui opposent partisans de sa disparition pure et simple comme Penty et ces Guild Socialists qui sont favorables à une forme de coopération avec l'État, tels S.G. Hobson. Pour davantage de précisions se reporter à l'article de Jean-Paul Révauger, «Socialisme et nostalgie : les Guild Socialists, émules de William Morris ", in Béatrice LAURENT (dir.), William Morris : News from Nowhere. Paris : Éditions du Temps, 2004.

32. La filiation avec Ruskin est incontournable ; les premières guildes s'établissent sur le modèle de la Guilde de St George.

33. News from Nowhere, op. cit., p. 77. 
34. On peut citer ici J. N. FIGGIS qui écrit: 'The State may recognize and guarantee...the life of these societies - the family, the club, the union, the college, the Church; but it no more creates that life than it creates the individual.' Churches in the Modern State, Londres, 1913, p. 224. Cité par W. H. GREENLEAF, The Ideological Heritage, p. 423.

35. Sur ces questions voir W. H. GREENLEAF, The Ideological Heritage, pp. 422-439.

36. Notons par exemple les progrès du syndicalisme révolutionnaire britannique qui milite en faveur d'une radicalisation de l'action syndicale. À ce propos, voir l'article de Jean-Paul Révauger, op. cit.

37. Noel THOMPSON, Political Economy and the Labour Party, p. 33.

\section{RÉSUMÉS}

William Morris appartient indiscutablement à la tradition socialiste britannique, mais lorsqu'il s'agit de définir la nature de la contribution d'un auteur aussi atypique à l'émergence d'une pensée socialiste en Grande-Bretagne, les certitudes s'estompent. C'est à partir d'une analyse du discours utopique de William Morris, des tensions qui opposèrent l'auteur de News from Nowhere aux grandes voix du socialisme naissant et d'une étude des prolongements de la pensée morrissienne dans les travaux des Guild Socialists qu'il sera possible d'en préciser les contours.

That William Morris belongs to the British socialist tradition is an indisputable fact, but should one decide to try and identify the nature of this eccentric author's contribution to the emergence of socialism in Great Britain, all certainties tend to fade away. This paper concentrates on Morris's use of utopia, on the many occasions when his views clashed with those of the senior members of the budding socialist movement and finally on the distinctively Morrissian hue of many of the Guild Socialists' publications. It will then be possible to bring William Morris's socialism into focus.

\section{AUTEUR}

\section{VALÉRIE AUDA-ANDRÉ}

Université de Provence : Aix-Marseille 